\title{
EFFECT OF SOME EXPERIMENTAL FACTORS ON PREPARATION OF LIQUID NATURAL RUBBER
}

\author{
Doan Minh Khai ${ }^{*}$, Phan Duc Nhan \\ Military Technical Academy, 236-Hoang Quoc Viet, Hanoi \\ *Email:khaihv@gmail.com
}

Received: 20 November 2015; Accepted for publication: 18 July 2016

\begin{abstract}
The article presents a study of effect of factors such as the types of irradiation, the concentration of NR solution and the content of hydrogen peroxide in mixture on photochemical depolymerization of natural rubber. The results showed that the ultraviolet irradiation from the vapor mercury lamps (HSW-250W and HSW-125W) caused the faster rate of depolymerization than the light from HF-500W; increasing concentration of NR in toluene leaded to decrease the rate of depolymerization; and as the content of hydrogen peroxide increased the rate of depolymerization was faster. Additionally, the IR spectra, ${ }^{13} \mathrm{C}-\mathrm{NMR}$ of products were recorded and showed that the cis-1,4-isoprene structure has not been changed and there was the presence of hydroxyl-terminated groups in chains.
\end{abstract}

Keywords: liquid natural rubber, photochemical method, molecular weight.

\section{INTRODUCTION}

Natural rubber (NR) mainly consists of cis-1,4-polyisoprene which is more than $92 \%$ in weight of NR [1]. Average molecular weight $(\bar{M})$ of NR is from several hundred thousand to about million. In this case, it cannot be used for polymer composites which either contain the power-intensive fillers or are formed by casting as a fuel-binder for solid rocket propellants. For this purpose, it is necessary to strongly decrease molecular weight of NR to below 50,000 [2].

Liquid natural rubber (LNR) can be synthesized by many methods including mechanic impact, thermal degradation, redox and photochemical method etc [1-8]. By those methods, the molecular weight of the LNRs was from 2,000 to 50,000. When the depolymerization of NR by mechanic impact and thermal degradation, the LNR contained the various functional groups. These processes were very difficult to control [1]. Pham Huu Ly et al. [4] synthesized LNR by redox method that used an agent $\mathrm{Fe}^{2+} / \mathrm{H}_{2} \mathrm{O}_{2}$ (Fenton method) and by Fenton including irradiation. They stated that the optimal conditions of reaction were: $\mathrm{pH} \sim 3$, ratio $\mathrm{H}_{2} \mathrm{O}_{2} / \mathrm{Fe}^{2+} \sim 1.5$, concentration of $\mathrm{NR} \sim 5 \%$ and temperature at $35^{\circ} \mathrm{C}$. Hereby, the average molecular weight of LNR was 4,500 after 80 hours and 3,800 after 100 hours [4]. 
For the photochemical method, Cumeen carried out photochemical degradation of NR by ultraviolet irradiation in the presence of nitrobenzene. By this method, the degraded LNR had an average molecular weight of 3,000 and contained carbonyl-terminated groups [1]. At Cochin University in India, Ravindran et al. [3] prepared hydroxyl-terminated poly-isoprene (HTPI) by photochemical method that used ultraviolet irradiation of lamp HPK-125W in the presence of hydrogen peroxide in organic solvents (toluene/methanol or toluene/tetra-hydro-furan). The obtained LNRs had average molecular weight from 8,100 to 30,000 and contained hydroxylterminated groups and a few epoxy groups [3]. Additionally, the effect of time period on average molecular weight of LNR was investigated. Herein, the average molecular weight of LNR was 20,400 after 20 hours and 15,100 after 30 hours.

In summary, the previous researches have not investigated the effects of lamp capacity, irradiation types, concentration of NR, neither the ratio of hydrogen peroxide to NR on the efficiency of photochemical degradation. Thus, in this study these factors are investigated.

\section{MATERIALS AND METHODS}

\subsection{Materials and apparatus}

- NR $(\bar{M}=820,000)$ was collected from latex in Binh Phuoc province; Hydrogen peroxide PA $30 \%$; Toluene PA; Methanol PA (XiLong, China).

- Apparatus: Flat bottom balloon $500 \mathrm{ml}$; magnetic stirrer; vapor mercury lamp $40 \mathrm{~W}$; Halogen floodlight HF-500W; vapor mercury lamp HSW-125W (wave-length $365 \mathrm{~nm}$, capacity $400 \mathrm{~mW} / \mathrm{m}^{2}$ ); vapor mercury lamp HSW-250W (wave-length $365 \mathrm{~nm}$, capacity 700 $\mathrm{mW} / \mathrm{m}^{2}$ ); vacuum oven; viscometer Ostwald $0.6 \mathrm{~mm}$.

\subsection{Preparation of $L N R$}

In a flat bottom balloon $500 \mathrm{ml}$, NR was dissolved in toluene within range of concentration from 3 to $10 \%(\mathrm{w} / \mathrm{v})$ by stirring. Then, the solution of NR in toluene was mixed with methanol in the volume ratio (toluene/methanol) 20:3. Hydrogen peroxide solution was added to the balloon in the ratio (hydrogen peroxide/NR) from 1:20 to 3:10. A mix was stirred and irradiated by the lamp. After interval time, a part of sample was collected, purified, dried, then was determined intrinsic viscosity and calculated average molecular weight. The sample was precipitated and purified by methanol/toluene three times. The sample was dried by vacuum oven at $65^{\circ} \mathrm{C}$ during 24 hours.

\subsection{Determination of intrinsic viscosity and calculation of average molecular weight}

Concentration of the sample in toluene was from $0.025 \%$ to $0.4 \%(\mathrm{w} / \mathrm{v})$. Viscosity of the samples was measured by viscometer Ostwald. Then, intrinsic viscosity of the sample was linearly extrapolated by curve between specific viscosity and concentration when the concentration is zero. The $\bar{M}$ of sample was calculated by equation as $[\eta]=K \cdot M_{v}^{a}$. Where: $[\eta]$ - intrinsic viscosity; $\mathrm{K}$, a - the constants depending on type of solvent, temperature; LNR is dissolved in toluene at $25^{\circ} \mathrm{C}: \mathrm{K}=9.6 \times 10^{-6}$; $\mathrm{a}=1.026$ [5].

\subsection{Fourier transform infrared spectroscopy (FTIR)}


FTIR of LNR was recorded by Perkin Elmer Spectrum 400 at Military Technical Academy. Pellets of $2 \mathrm{mg}$ of LNRs were prepared by mixing with $200 \mathrm{mg}$ of spectroscopic grade $\mathrm{KBr}$. IR spectra $\left(4000-400 \mathrm{~cm}^{-1}\right)$ were recorded with a resolution of $4 \mathrm{~cm}^{-1}$ and 32 scans per sample.

\subsection{Method of ${ }^{13} \mathrm{C}-\mathrm{NMR}$ spectra}

${ }^{13} \mathrm{C}-\mathrm{NMR}$ spectra of $\mathrm{LNR}$ in $\mathrm{CDCl}_{3}$ was recorded at $300^{\circ} \mathrm{K}$ by Bruker equipment, at the laboratory of Vietnam National University, Hanoi.

\section{RESULTS AND DISCUSSION}

\subsection{Effect of the types of irradiation on efficiency of degradation}

For process of photochemical degradation of NR, type of irradiation is effect on forming free radical agents. The agents are formed by decomposing hydrogen peroxide with irradiations in the range from $600 \mathrm{~nm}$ to $300 \mathrm{~nm}$ (energy from 200 to $400 \mathrm{~kJ} / \mathrm{mole}$, respectively) [1]. The higher concentration of free radial the higher rate of degradation. In this work, various irradiated types were chosen (Table 1). In each experiment, the concentration of NR in toluene was fixed at $5 \%(\mathrm{w} / \mathrm{v})$ and the ratio of components (toluene/methanol/hydrogen peroxide) was fixed at 20:3:1 [3,8]. The results are shown in Table 1.

Table 1. Effect of irradiation types on intrinsic viscosity and average molecular weight.

\begin{tabular}{|c|l|c|c|c|}
\hline \multirow{2}{*}{ No. } & \multicolumn{1}{|c|}{ Types of irradiation } & \multirow{2}{*}{$\begin{array}{c}\text { Time of photochemical } \\
\text { degradation }\end{array}$} & \multicolumn{2}{|c|}{ Characteristics of LNR } \\
\cline { 4 - 5 } & & {$[\eta]$} & $\bar{M}_{v}$ \\
\hline 1 & Mercury vapor lamp UVA-40W & 15 days & 0.72 & 56,400 \\
\hline 2 & Halogen floodlight HF-500W & 22 hours & 1.21 & 93,600 \\
\hline 3 & Mercury vapor lamp HSW-125 W & 22 hours & 0.25 & 20,100 \\
\hline 4 & Mercury vapor lamp HSW-250W & 22 hours & 0.19 & 15,400 \\
\hline
\end{tabular}

The results in Table 1 show that when the capacity of HSW-125W lamp is about 3 times higher than that of UVA-40W lamp, the efficiency of the HSW-125W lamp is increased so that: the time of degradation is decreased of about 16 times but the average molecular weight is still decreased of 2.8 times. Comparing the HF-500W lamp with the mercury vapor HSW$125 \mathrm{~W}$ lamp, while either the time is the same ( 22 hours) and the capacity is higher (about 4 times), molecular weight from the first is still about 4.6 times as many as with the HSW-125W lamp. Compared with the others, the HSW-250W lamp has the highest efficiency.

In summary, when increasing capacity of the irradiating lamps, intrinsic viscosity and molecular weight of the LNR and time of photochemical degradation are decreased.

\subsection{Effect of ratio of hydrogen peroxide to NR on molecular weight of LNR}

De-polymerization of NR depends on not only the types and capacity of irradiation but also a ratio of component of the reactive mixing. In the mix, hydrogen peroxide is not 
dissolved in the NR solution. An amount of methanol is necessary for homogenization of the mixture. The suitable ratio of toluene to methanol is 20:3 [1,8]. In this paper, in order to investigate effect of NR solution in toluene and the ratio of $\mathrm{H}_{2} \mathrm{O}_{2}$ to NR the following conditions were chosen: concentration of NR in toluene in the range from 3 to $10 \%$; ratio of $\mathrm{H}_{2} \mathrm{O}_{2}$ to NR 0.5:10, 1:10, 3:10; the HSW-250W lamp; time of reaction 22 hours. The results are shown in Table 2.

Table 2. Effect of NR solution in toluene and the ratio of $\mathrm{H}_{2} \mathrm{O}_{2}$ to NR on molecular weight.

\begin{tabular}{|c|c|c|c|c|}
\hline \multirow{2}{*}{$\begin{array}{c}\text { The } \\
\text { samples }\end{array}$} & Concentration of NR, \% & \multirow{2}{*}{$\begin{array}{c}\text { Ratio of toluene/methanol/hydrogen } \\
\text { peroxide }\end{array}$} & \multicolumn{2}{|c|}{ Characteristics of LNR } \\
\cline { 4 - 5 } & & $20: 3: 1$ & {$[\eta]$} & $\bar{M}_{v}$ \\
\hline LNR-1 & 3 & $20: 3: 1$ & 0.17 & 13,800 \\
\hline LNR-2 & 5 & $20: 3: 1$ & 0.19 & 15,400 \\
\hline LNR-3 & 10 & $20: 3: 0.5$ & 0.25 & 20,100 \\
\hline LNR-4 & 10 & $20: 3: 3$ & 0.29 & 23,300 \\
\hline LNR-5 & 10 & & 0.17 & 13,800 \\
\hline
\end{tabular}

It is shown in Table 2 that when increasing concentration of NR from 3 to $10 \%$, the $\bar{M}$ of LNR is up. It can be explained by two reasons: firstly, the interaction of hydrogen peroxide with NR is decreased because viscosity of the mixture is increased; secondly, the ratio of hydrogen peroxide to NR is down (from 10:3 to 10:1). However, the $\bar{M}$ of LNR at concentration of $10 \%$ NR $(20,100)$ is about 1.5 times as many as $3 \%(13,800)$ while the yield of an experiment is up about 3.3 times. When continue increasing the photochemical time, the $\bar{M}$ of LNR can still be reduced. This problem is necessary of interest to study in larger scale.

The results in Table 2 show that when increasing the ratio of hydrogen peroxide to NR the molecular weight of LNR is down. The molecular weight is down from 23,300 to 13,800 when the ratio is up by 6 times. Additionally, the molecular weight of either LNR-1 sample and LNR5 sample is the same, herein the ratio of hydrogen peroxide is $1: 3$. It is indicated that the ratio of hydrogen peroxide to NR is decidedly effecting on molecular weight of LNR.

\subsection{Effect of photochemical time on molecular weight of LNR}

The photochemical time also affects molecular weight of LNR. In order to investigate effect of the time, an experimental attempt was carried out in the range from 7 to 40 hours, at concentrations of $3 \%, 5 \%$ and $10 \%$ NR, using HSW-250W lamp. The results are shown in Table 3.

Table 3. The data of effect of the time on molecular weight of LNR.

\begin{tabular}{|c|c|c|c|c|}
\hline \multirow{2}{*}{ No. } & \multirow{2}{*}{$\begin{array}{c}\text { Photochemical time, } \\
\text { hours }\end{array}$} & $3 \%$ & $5 \%$ & $10 \%$ \\
\cline { 3 - 5 } & 7 & 30,600 & 39,000 & 72,000 \\
\hline 1 & 14 & 19,200 & 25,300 & 37,500 \\
\hline 2 & 22 & 13,800 & 15,400 & 20,100 \\
\hline 3 & 30 & 7,150 & 8,700 & 10,600 \\
\hline 4 & 40 & 6,420 & 7,500 & 8,200 \\
\hline 5 & & & & \\
\hline
\end{tabular}


As shown in the Table 3, the $\bar{M}$ of LNR strongly depends on the time and the concentration of NR in toluene. In three cases, when increasing the time the $\bar{M}$ of LNR is down. Especially, at the first 30 hours the $\bar{M}$ is decreased more quickly. Then, at the later time the rate of decrease of $\bar{M}$ is slow down. In other case, when increasing concentration of NR in toluene, at the first time period the molecular weight of LNRs is much different. But, when the time is up to 40 hours, the $\bar{M}$ of the LNRs is not much different.

\subsection{Investigation of the characteristics of LNR}

The photochemical degradation of NR by oxidant agent causes de-polymerization of NR chain and addition of functional groups to chain. In this work, in order to investigate the change of LNR from NR structure, it was carried out recording FTIR spectra and ${ }^{13} \mathrm{C}-\mathrm{NMR}$ spectra of LNR. The results are shown in Figure 1 and Figure 2.

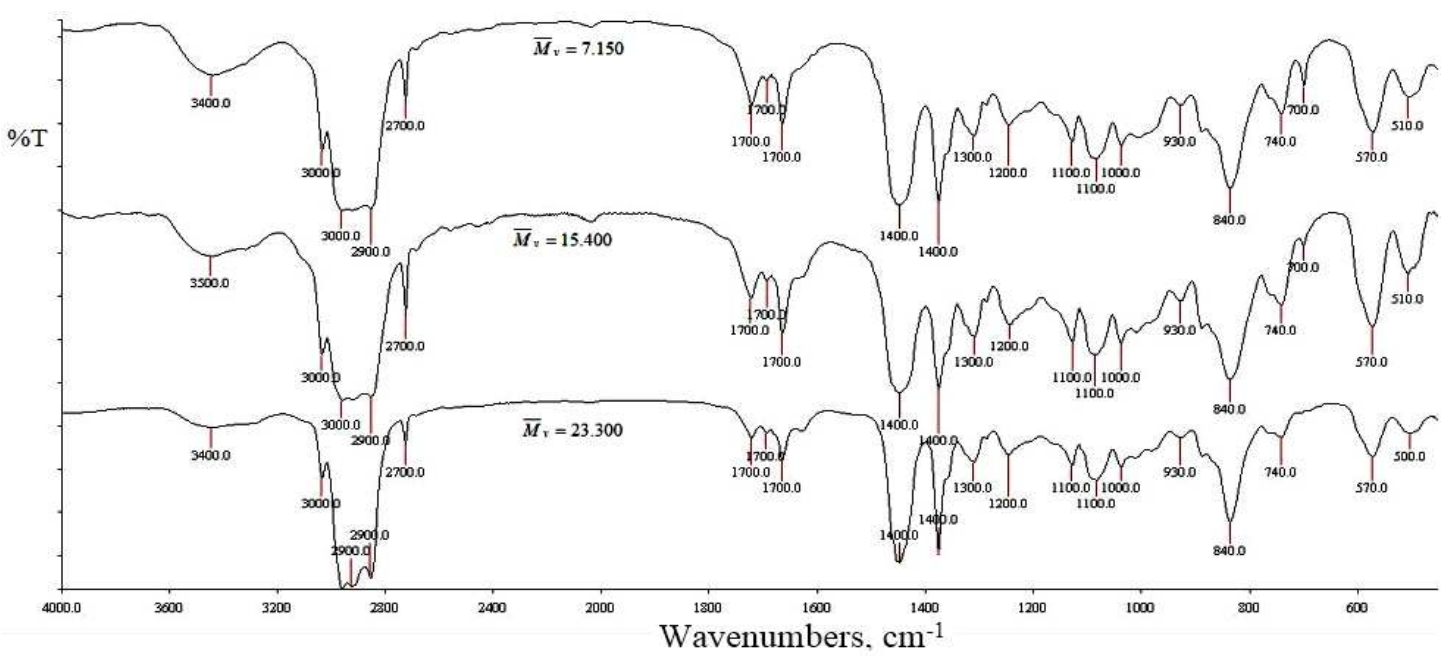

Figure 1. FTIR spectra of the LNR samples.

FTIR spectra of LNR samples in Figure 1 show that the bands at $840 \mathrm{~cm}^{-1}$ and 1660 $\mathrm{cm}^{-1}$ are of $\mathrm{C}-\mathrm{H}$ and $\mathrm{C}=\mathrm{C}$ in cis-vinylene groups $(>\mathrm{C}=\mathrm{CH}-)$, respectively. The bands at $1375 \mathrm{~cm}^{-1}, 1450 \mathrm{~cm}^{-1}$ and $2800 \div 3000 \mathrm{~cm}^{-1}$ are of $\mathrm{C}-\mathrm{H}$ in methyl and methylene $\left(-\mathrm{CH}_{3}\right.$, $\mathrm{CH}_{2}$ ). Especially, the presence of the band at $3400 \mathrm{~cm}^{-1}$ indicates functional hydroxyl groups of LNR chains. Additionally, the the number of the hydroxyl groups are increased when the $\bar{M}$ of LNR is down. From the above mentioned two factors, it can be revealed that the molecular of LNRs contained hydroxyl-terminated groups. 

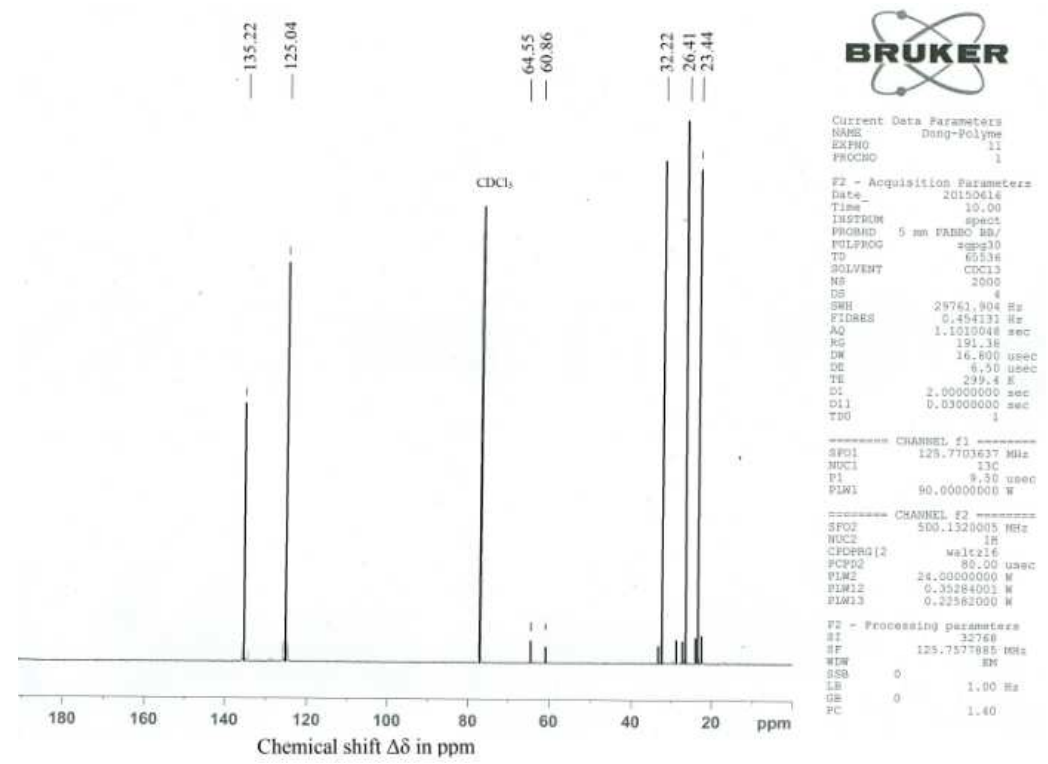

Figure 2. ${ }^{13} \mathrm{C}-\mathrm{NMR}$ spectra of LNR sample in $\mathrm{CDCl}_{3}$ at $300 \mathrm{~K}\left(\bar{M}_{v}=7150\right)$.

As shown in Figure 2, the peak of chemical shift $(\Delta \delta)$ at $23.44 \mathrm{ppm}$ is of methyl $\left(-\mathrm{CH}_{3}\right)$. The peaks of $\Delta \delta$ at $26.41 \mathrm{ppm}$ and $32.22 \mathrm{ppm}$ are of methylene $\left(-\mathrm{CH}_{2}\right)$; and the peaks of chemical shift at $125.04 \mathrm{ppm}$ and $135.22 \mathrm{ppm}$ are of cis-vinylene $(>C=C<)$ in cis-1,4isoprene units. Especially, the presence of two peaks at $60.86 \mathrm{ppm}$ and $64.55 \mathrm{ppm}$ are of carbon atoms of $-\mathrm{CH}_{2}-\mathrm{OH}$ and $>\mathrm{CH}-\mathrm{OH}$ groups, respectively. Furthermore, the weak peaks of chemical shift in the range from 21 to $24 \mathrm{ppm}$ are of ethyl and methylene at terminal of LNR chain.

In summary, the photochemical degradation of NR is either decrease of $\bar{M}$ or not change of chain structure, as well as addition of hydroxyl-terminated groups. Hence, these LNRs have useful properties for fuel-binder of solid rocket propellant.

\section{CONCLUSION}

The $\bar{M}$ of LNR, which is prepared by photochemical method, depends on type and capacity of irradiation. Among the used lamps, the efficiency of HSW-250 lamp is much better than those of the others. The average molecular weight also depends on concentration of NR in toluene and ratio of hydrogen peroxide to NR. The efficiency of degradation is optimal when the concentration is of $10 \% \mathrm{NR}$ and the ratio of hydrogen peroxide to NR is of 3:1. Additionally, the photochemical time also is used to control $\bar{M}$ of LNR. The IR spectra and ${ }^{13} \mathrm{C}-\mathrm{NMR}$ spectra of LNR confirm that the LNR samples do not mostly change structure of cis-1,4-isoprene units and indicate the presence of hydroxyl-terminated groups.

\section{REFERENCES}

1. Husin Mohd Nor, John R. - Telechelic liquid natural rubber: A review, Pro, Polym. Sci. 23 (1998) 143-177. 
2. Norfhairna Baharulrazi et al. - Hydroxyl terminated natural rubber as a binder in solid rocket propellant, Applied Mechanics and Materials, Trans Tech Publication Ltd, 695 (2015) 174-178.

3. Thottakkat Ravindran et al. - A novel method for the preparation of hydroxyl terminated liquid natural rubber, Makromol. Chem., Rapid Commun. 7 (1986) 159-163.

4. Phạm Hữu Lý và cộng sự - Tổng hợp cao su thiên nhiên lỏng có nhóm hydroxyl cuối mạch bằng phương pháp fenton quang hóa: Nghiên cứu ảnh hưởng của các điều kiện phản ứng fenton quang hóa, Tạp chí Khoa học và Công nghệ 49 (6) (2011) 93-100.

5. Siti Zaleha Isa et al. - The influence of temperature and reaction time in the degradation of natural rubber latex, The Malaysian Journal of Analytical Sciences 11 (1) (2007) 42-47.

6. Yasuyuki Tanaka et al. - Depolimerized natural rubber and process for preparation thereof, United States Patent No.5,622,998 (1997).

7. Ekasit Anancharoenwong - Synthesis and characterization of cis-1,4-polyisoprene-based polyurethane coatings; study of their adhesive properties on metal surface, The $\mathrm{PhD}$ thesis of University of Maine Thailand, 2011, pp. 53-75.

8. Ghiggino K.P. - Progress in Pacific Polymer Science 3, Proceedings of the Third Pacific Polymer Conference Gold Coast, Queensland, December 13-17, 1993, Springer-Verlag Berlin Heidelberg, 1994, pp. 351-366. 